\title{
Sequência de ossificação do sincrânio e hioide em embriões de Caiman yacare (Crocodylia, Alligatoridae)
}

\author{
Fabiano C. Lima ${ }^{1}$; André Luiz Q. Santos ${ }^{2}$; Lucélia G. Vieira ${ }^{3}$ \& Marcos E. Coutinho ${ }^{4}$
} 1. Laboratório de Anatomia Humana e Comparativa, Universidade Federal de Goiás. Rua José de Carvalho Bastos, 491, Centro, 75800-047, Jataí, GO, Brasil. (fabianocl21@hotmail.com)
2. Laboratório de Ensino e Pesquisa em Animais Silvestres - LAPAS, Faculdade de Medicina Veterinária da Universidade Federal de Uberlândia. Av. Amazonas 2245, Jardim Umuarama,
38400-000, Uberlândia, MG, Brasil. (quagliatto@famev.ufu.br)
3. Laboratório de Anatomia Comparativa dos Vertebrados, Universidade de Brasília. SHCGN 707 Bloco C apto. 113, Asa Norte, 70730-730, Brasília, DF, Brasil. (luceliabio@yahoo.com.br)
4. Instituto Chico Mendes para Conservação da Biodiversidade, Centro de Conservação e Proteção de Répteis e Anfibios. Av. Vilma Edelweiss Santos, 115, 33400-00, Lagoa Santa, MG, Brasil.
(lapas_bio@yahoo.com.br)

\begin{abstract}
Ossification sequence of skull and hyoid in embryos of Caiman yacare (Crocodylia, Alligatoridae). The skull is a unique and complex structure of vertebrates and a main focus of morphological and systematic studies. Although Crocodilians represent an important component of Archosauria group, our knowledge of the development and homology are few. Herein, we describe the detailed development sequence of skull of the Caiman yacare (Daudin, 1802) in an effort to contribute to filling this anatomical gap. Embryos were sampled randomly at a regular intervals during full incubation period and morphological descriptions are based on cleared and stained and embryos. The ossification pattern of C. yacare follow general aspects for reptiles and others tetrapods. The first ossification centers occur in dermal bones involved in primary functions such as feeding and breathing, like maxillae, dentary, splenial, angular, pterygoid, ectopterygoid and jugal, including the teeth. Bones of dorsal portion of the neurocraniun ossify later, showing a cranial fontanelle that remains at the hatching. The parietal, frontal and opisthotic have more than one ossification center that fuse during ontogeny. The ossification center for parasphenoid was absent and only one ossification center is present for basisphenoid bone. The posterior portion of the skull is formed for ossification centers of chondrocranium substitution that ossify in later stages.
\end{abstract}

KEYWORDS. Morphology, ontogeny, ossification, Pantanal caiman, reptiles.

RESUMO. O crânio representa uma estrutura única e complexa dos vertebrados, sendo foco relevante objeto de estudos morfológicos e sistemáticos. Embora os crocodilianos constituam um importante grupo representante dos Archosauria, nossos conhecimentos acerca de seu desenvolvimento e homologias ainda são escassos. Aqui descrevemos uma sequência detalhada de ossificação dos ossos do crânio de Caiman yacare (Daudin, 1802), objetivando contribuir com informações de foco anatômico. Coletaram-se ao acaso embriões em intervalos regulares durante todo o período de incubação, sendo estes posteriormente submetidos a protocolo de diafanização e coloração de ossos. O padrão de ossificação em C. yacare segue parâmetros gerais em répteis e outros tetrápodes. Os primeiros centros de ossificação correspondem aos ossos dérmicos, envolvidos com funções primárias como a alimentação e respiração (e.g. maxila, dentário, esplenial, angular, pterigoide, ectopterigoide e jugal, incluindo ainda os dentes). Os ossos da porção dorsal do neurocrânio se ossificam posteriormente, evidenciando uma fontanela cranial que permanece até o momento da eclosão. Os ossos parietal, frontal e opstótico possuem mais de um centro de ossificação que se fundem durante a ontogenia. O centro de ossificação do parisfenoide está ausente, e apenas um centro de ossificação está presente para o basisfenoide. A porção posterior do crânio é formada por centros de substituição do condrocrânio que se ossificam em estágios posteriores.

PALAVRAS-CHAVE. Morfologia, ontogenia, ossificação, jacaré-do-pantanal, répteis.

Dentre os elementos do esqueleto, o crânio é um foco importante para estudos de morfologia e sistemática. Embora exista uma considerável variedade de informações acerca deste componente anatômico para variadas espécies, suas mudanças estruturais e o desenvolvimento ainda são relativamente pouco conhecidos (DE SÁ, 1988). A presença da cabeça formada por esqueleto ósseo é postulado como a diferença mais conspícua entre os cordados e os protocordados (GANS \& NorThCUTT, 1983). O desenvolvimento desta estrutura deriva de migrações das células da crista neural, que sob influência de fatores gênicos produzem condensações que formarão os ossos do crânio (GANS \& NORTHCUTT, 1983; NorthCutt \& Gans, 1983; Langille \& Hall, 1987).

Os diferentes padrões de crescimento observados no esqueleto dos vertebrados são promovidos durante a morfogênese, mas anteriormente determinados por um conjunto de processos morfogenéticos. Esta dinâmica pode refletir em fatores ambientais, mecânicos ou funcionais e estimular ou inibir o crescimento, especialmente no crânio (Herring, 1993; Richtsmeier et al., 1993; Monteiro \& SOARES, 1997; Monteiro et al., 1997).
Em alguns casos, mudanças drásticas na morfologia de alguma estrutura (e.g. a redução do número de dígitos Alberch et al., 1979, ou como o desenvolvimento do casco dos testudines BuRKE, 1989) podem ser o resultado de mecanismos simples de desenvolvimento (YeH, 2002). Outro exemplo são os períodos de ossificação dos diversos ossos do crânio em diferentes vertebrados. As variações mais significativas refletem aquisições funcionais de cada espécie, e uma vez necessárias à sobrevivência da mesma, são conservadas ao longo de sua história de vida, mesmo que com pequenas adaptações (LANGILLE \& Hall, 1987; Yen, 2002). Diferenças no tempo relativo de ossificação podem indicar processos de heterocronia, uma importante característica no desenvolvimento e evolução do esqueleto (HANKEN \& HALL, 1988).

Os répteis representam uma diversidade notável de espécies encontradas em diversos habitats distribuídos pelo planeta. Estão divididos em quatro ordens: Testudines (tartarugas, cágados e jabutis), Lepidosauria (lagartos, serpentes, anfisbenias e tuataras), Crocodylia (crocodilos, jacarés e gaviais) e Aves (Rieppel \& ReIsz, 
1999; Schoch, 2006). Todavia a relação evolutiva dos Testudines para outros amniotas permanece controversa (Rieppel, 1995, 2004; Rieppel \& De Braga, 1996; Meyer \& Zardoya, 2003; Müller, 2003; Werneburg \& SANCHEZ-VILLAGRA, 2009).

Os crocodilianos incluem três subfamílias que abrangem 23 espécies de reconhecidas (BELLAIRS, 1987; Brochu, 2003; SBH, 2010). A maioria das espécies viventes encontram-se nas regiões tropicais e subtropicais, com alguns exemplares dispersos por regiões temperadas (NAISH, 2001; HiCKMAN et al., 2006; MAdER, 2006). Todos os crocodilianos viventes são caracterizados pelo bom desenvolvimento do esqueleto dérmico do crânio. Contudo, pouco se conhece sobre os processos ontogênicos que levam ao desenvolvimento do esqueleto dos crocodilianos [e.g. Alligator mississippiensis (Daudin, 1802), MüLlER \& AlBERCH, 1990, RiepPel, 1993; Alligator sinensis Fauvel, 1879, Mook, 1923; Crocodylus palustris Lesson, 1831, PARKER, 1882, 1883; Crocodylus niloticus Laurenti, 1768, IORDANSKY, 1973; Crocodylus porosus Schneider, 1801, MooK, 1921, KäLIN, 1933].

As características anatômicas referentes ao desenvolvimento do esqueleto dos crocodilianos são de interesse para discussões acerca dos padrões filogenéticos dos répteis (incluindo aves), bem como para análises estruturais ou biomecânicas (PARKER, 1883; MEEK, 1911; Mook, 1921, 1923; De Beer, 1937; Iordansky, 1973; Dodson, 1975; Müller \& Alberch, 1990; Rieppel, 1993; Klembara, 2004, 2005). Neste contexto, objetivase descrever a morfologia e a sequência detalhada da ossificação craniana em embriões de Caiman yacare (Daudin, 1802), no escopo de contribuir para o entendimento da filogenia dos Archosauria.

\section{MATERIAL E MÉTODOS}

Coleta, descrição morfológica e processamento de embriões. Ovos de C. yacare adquiridos na área de reprodução da Fazenda Pousada das Araras, Aquidauana, Mato Grosso do Sul, sob licença número 021/2007 IBAMA/RAN foram mantidos em sistema de incubação semi-artificial com temperatura média de $32,3^{\circ} \mathrm{C}\left( \pm 0,5^{\circ} \mathrm{C}\right)$. Os embriões foram removidos de seus ovos em intervalos regulares de três dias, o que permitiu manter a sequência ontogenética desde o primeiro dia de incubação natural até sua eclosão. Cada espécime foi fixado em solução de formol 10\% e encaminhado ao Laboratório de Ensino e Pesquisa em Animais Silvestres - LAPAS da Universidade Federal de Uberlândia. Posteriormente foi realizada a diafanização por hidróxido de potássio $(\mathrm{KOH} 2 \%)$ e coloração dos ossos por vermelho de alizarina, conservando o material em glicerina segundo o método de DAvis \& GoRE (1936). A estrutura geral do crânio cartilaginoso de algumas espécies de crocodilianos é relativamente bem descrita na literatura (SHIINO, 1914; BELAIRS \& KAMAL,
1981; Klembara, 1991, 2005). Outrora, devido algumas dificuldades na coleta, foi inviável conduzir e relatar as etapas de formação do condrocrânio em C. yacare.

A presença de centros de ossificação foi analisada em microscópio estereoscópico acoplado a um sistema de captura de imagens. A investigação foi autorizada pelo Comitê de Ética em Pesquisas Animais da Universidade Federal de Uberlândia sob protocolo CEUA032/2009. Os embriões utilizados estão depositados no acervo do LAPAS sob os registros LD07Cy01/02 a LD07Cy57/02. São descritos os espécimes LD07Cy30/02 (30 dias), LD07Cy33/02 (33 dias), LD07Cy36/02 (36 dias), LD07Cy39/02 (39 dias), LD07Cy42/02 (42 dias), LD07Cy45/02 (45 dias), LD07Cy48/02 (48 dias), LD07Cy51/02 (51 dias), LD07Cy54/02 (54 dias) e LD07Cy57/02 (57 dias).

Ossificação e sequência ontogenética. De maneira geral, a retenção de vermelho de alizarina nos ossos dos embriões foi excelente. No entanto, a formação óssea precoce nos estágios iniciais não foi indicada pela coloração em alguns exemplares, mas sim pela textura diferenciada da estrutura óssea (RIEPPEL, 1993; SHeIL, 2005). Para a comparação do padrão de ossificação através das espécies aqui apresentadas, apenas a sequência relativa foi importante. Os trabalhos de Ferguson (1985, 1987), Magnusson \& TAYlor (1980) e IUNGMAN et al. (2008) servirão de subsídios, sendo entretanto consideradas diferenças inter-específicas nos tempos de incubação (DE SÁ, 1988).

Os espécimes foram examinados e a presença ou ausência de cada um dos elementos ósseos foi registrado para determinar a sequência ontogenética dos eventos durante a ossificação. Comparamos os dados obtidos para C. yacare com outros grupos além de Archosauria, como Lepidosauria e Testudines. As descrições morfológicas, bem como a nomenclatura dos ossos do crânio seguem RoMer (1956), IORDANSKy (1973) e RiEPPEL (1993).

\section{RESULTADOS}

Os primeiros registros de retenção de corante foram feitos a partir do dia 30, como relatado, mas toda a sequência foi avaliada. Foi produzida uma tabela com a sequência de ossificação, abordando separadamente os ossos de formação dérmica e endocondral (Tab. I).

Pré-maxila. É visualizado no início do desenvolvimento como um elemento não corado na porção rostral do focinho, aos 33 dias, sobre a cápsula nasal. Este elemento está bem corado aos 36 dias. Ambos os centros de ossificação da pré-maxila não se contatam até o fim do período de incubação. Surge como uma pequena placa orientada transversalmente entre a placa parietotectal e a lâmina transversa anterior. Com 51 dias ele contata o nasal e depois a maxila e seu colateral (Figs 1-12).

Maxila. É um dos primeiros ossos visíveis no embrião de 30 dias, como uma placa triangular, pequena 
Tabela I. Resumo da sequência de ossificação do sincrânio de Caiman yacare (Daudin, 1802).

\begin{tabular}{|c|c|c|c|c|c|c|c|c|c|c|}
\hline \multicolumn{11}{|c|}{ Ossos Dérmicos } \\
\hline & \multicolumn{10}{|c|}{ Dias de incubação } \\
\hline & 30 & 33 & 36 & 39 & 42 & 45 & 48 & 51 & 54 & 57 \\
\hline Pré-maxila & & $\mathrm{X}$ & $\mathrm{x}$ & $\mathrm{x}$ & $\mathrm{X}$ & $\mathrm{x}$ & $\mathrm{x}$ & $\mathrm{x}$ & $\mathrm{x}$ & $\mathrm{x}$ \\
\hline Maxila & $\mathrm{x}$ & $\mathrm{x}$ & $\mathrm{x}$ & $\mathrm{x}$ & $\mathrm{x}$ & $\mathrm{x}$ & $\mathrm{x}$ & $\mathrm{x}$ & $\mathrm{x}$ & $\mathrm{x}$ \\
\hline Jugal & $\mathrm{X}$ & $\mathrm{x}$ & $\mathrm{x}$ & $\mathrm{x}$ & $\mathrm{X}$ & $\mathrm{X}$ & $\mathrm{x}$ & $\mathrm{x}$ & $\mathrm{x}$ & $\mathrm{x}$ \\
\hline Quadradojugal & & $\mathrm{x}$ & $\mathrm{x}$ & $\mathrm{x}$ & $\mathrm{x}$ & $\mathrm{x}$ & $\mathrm{x}$ & $\mathrm{x}$ & $\mathrm{x}$ & $\mathrm{x}$ \\
\hline Pós-orbital & $\mathrm{x}$ & $\mathrm{x}$ & $\mathrm{x}$ & $\mathrm{x}$ & $\mathrm{x}$ & $\mathrm{x}$ & $\mathrm{x}$ & $\mathrm{x}$ & $\mathrm{x}$ & $\mathrm{x}$ \\
\hline Lacrimal & & $\mathrm{x}$ & $\mathrm{x}$ & $\mathrm{x}$ & $\mathrm{x}$ & $\mathrm{x}$ & $\mathrm{x}$ & $\mathrm{x}$ & $\mathrm{x}$ & $\mathrm{x}$ \\
\hline Pré-frontal & & $\mathrm{x}$ & $\mathrm{x}$ & $\mathrm{x}$ & $\mathrm{x}$ & $\mathrm{x}$ & $\mathrm{x}$ & $\mathrm{x}$ & $\mathrm{x}$ & $\mathrm{x}$ \\
\hline Frontal & & & $\mathrm{x}$ & $\mathrm{x}$ & $\mathrm{x}$ & $\mathrm{x}$ & $\mathrm{x}$ & $\mathrm{x}$ & $\mathrm{x}$ & $\mathrm{x}$ \\
\hline Esquamosal & & $\mathrm{x}$ & $\mathrm{x}$ & $\mathrm{x}$ & $\mathrm{x}$ & $\mathrm{x}$ & $\mathrm{x}$ & $\mathrm{x}$ & $\mathrm{x}$ & $\mathrm{x}$ \\
\hline Parietal & & & $\mathrm{x}$ & $\mathrm{x}$ & $\mathrm{x}$ & $\mathrm{x}$ & $\mathrm{x}$ & $\mathrm{x}$ & $\mathrm{x}$ & $\mathrm{x}$ \\
\hline Palatino & & $\mathrm{x}$ & $\mathrm{x}$ & $\mathrm{x}$ & $\mathrm{x}$ & $\mathrm{x}$ & $\mathrm{x}$ & $\mathrm{x}$ & $\mathrm{x}$ & $\mathrm{x}$ \\
\hline Vômer & & $\mathrm{x}$ & $\mathrm{x}$ & $\mathrm{x}$ & $\mathrm{x}$ & $\mathrm{x}$ & $\mathrm{x}$ & $\mathrm{x}$ & $\mathrm{x}$ & $\mathrm{x}$ \\
\hline Ectopterigóide & & & $\mathrm{x}$ & $\mathrm{x}$ & $\mathrm{x}$ & $\mathrm{x}$ & $\mathrm{x}$ & $\mathrm{x}$ & $\mathrm{x}$ & $\mathrm{x}$ \\
\hline Pterigóide & & $\mathrm{x}$ & $\mathrm{x}$ & $\mathrm{x}$ & $\mathrm{x}$ & $\mathrm{x}$ & $\mathrm{x}$ & $\mathrm{x}$ & $\mathrm{x}$ & $\mathrm{x}$ \\
\hline Dentário & $\mathrm{x}$ & $\mathrm{x}$ & $\mathrm{x}$ & $\mathrm{x}$ & $\mathrm{x}$ & $\mathrm{x}$ & $\mathrm{x}$ & $\mathrm{x}$ & $\mathrm{x}$ & $\mathrm{x}$ \\
\hline Esplenial & & $\mathrm{x}$ & $\mathrm{x}$ & $\mathrm{x}$ & $\mathrm{x}$ & $\mathrm{x}$ & $\mathrm{x}$ & $\mathrm{x}$ & $\mathrm{x}$ & $\mathrm{x}$ \\
\hline Suprangular & & & $\mathrm{x}$ & $\mathrm{x}$ & $\mathrm{X}$ & $\mathrm{X}$ & $\mathrm{x}$ & $\mathrm{x}$ & $\mathrm{x}$ & $\mathrm{x}$ \\
\hline Coronóide & & & & $\mathrm{x}$ & $\mathrm{x}$ & $\mathrm{x}$ & $\mathrm{x}$ & $\mathrm{x}$ & $\mathrm{x}$ & $\mathrm{x}$ \\
\hline Angular & & $\mathrm{X}$ & $\mathrm{x}$ & $\mathrm{x}$ & $\mathrm{X}$ & $\mathrm{x}$ & $\mathrm{x}$ & $\mathrm{x}$ & $\mathrm{x}$ & $\mathrm{x}$ \\
\hline Palpebral & & & & & & & $\mathrm{x}$ & $\mathrm{x}$ & $\mathrm{X}$ & $\mathrm{x}$ \\
\hline \multicolumn{11}{|c|}{ Ossos Endocondrais } \\
\hline & \multicolumn{10}{|c|}{ Dias de incubação } \\
\hline & 30 & 33 & 36 & 39 & 42 & 45 & 48 & 51 & 54 & 57 \\
\hline Quadrado & & & & $\mathrm{x}$ & $\mathrm{x}$ & $\mathrm{X}$ & $\mathrm{x}$ & $\mathrm{x}$ & $\mathrm{x}$ & $\mathrm{x}$ \\
\hline Basisfenóide & & $\mathrm{X}$ & $\mathrm{x}$ & $\mathrm{x}$ & $\mathrm{x}$ & $\mathrm{X}$ & $\mathrm{x}$ & $\mathrm{x}$ & $\mathrm{x}$ & $\mathrm{x}$ \\
\hline Lateroesfenóide & & & $\mathrm{x}$ & $\mathrm{x}$ & $\mathrm{x}$ & $\mathrm{x}$ & $\mathrm{x}$ & $\mathrm{x}$ & $\mathrm{x}$ & $\mathrm{x}$ \\
\hline Basioccipital & & $\mathrm{x}$ & $\mathrm{x}$ & $\mathrm{x}$ & $\mathrm{x}$ & $\mathrm{x}$ & $\mathrm{x}$ & $\mathrm{x}$ & $\mathrm{x}$ & $\mathrm{x}$ \\
\hline Exoccipital & & & & $\mathrm{x}$ & $\mathrm{X}$ & $\mathrm{X}$ & $\mathrm{x}$ & $\mathrm{x}$ & $\mathrm{x}$ & $\mathrm{x}$ \\
\hline Pró-ótico & & & & $\mathrm{x}$ & $\mathrm{x}$ & $\mathrm{x}$ & $\mathrm{x}$ & $\mathrm{x}$ & $\mathrm{x}$ & $\mathrm{x}$ \\
\hline Epi-ótico & & & & $\mathrm{x}$ & $\mathrm{x}$ & $\mathrm{x}$ & $\mathrm{x}$ & $\mathrm{x}$ & $\mathrm{x}$ & $\mathrm{x}$ \\
\hline Opstótico & & & & $\mathrm{x}$ & $\mathrm{x}$ & $\mathrm{x}$ & $\mathrm{x}$ & $\mathrm{x}$ & $\mathrm{x}$ & $\mathrm{x}$ \\
\hline Supraoccipital & & & $\mathrm{x}$ & $\mathrm{x}$ & $\mathrm{x}$ & $\mathrm{x}$ & $\mathrm{x}$ & $\mathrm{x}$ & $\mathrm{x}$ & $\mathrm{x}$ \\
\hline Articular & & & & $\mathrm{x}$ & $\mathrm{x}$ & $\mathrm{x}$ & $\mathrm{x}$ & $\mathrm{x}$ & $\mathrm{x}$ & $\mathrm{x}$ \\
\hline Hióide & & & $\mathrm{x}$ & $\mathrm{x}$ & $\mathrm{x}$ & $\mathrm{x}$ & $\mathrm{x}$ & $\mathrm{x}$ & $\mathrm{x}$ & $\mathrm{x}$ \\
\hline
\end{tabular}

e alongada, sem retenção de vermelho de alizarina, mas com a textura diferenciada das demais estruturas. Com 33 dias apresenta considerável retenção de corante, estando seu formato triangular conspícuo. A extremidade rostral é ampla e a posterior alongada, contatando pouco o jugal. A margem ventral da maxila forma a maior porção da margem labial superior. Com 42 dias, alguns dentes estão marcados por vermelho de alizarina, mas a maxila ainda não contata os ossos nasal e pré-maxila. Com 51 dias, está bem ossificada. Aos 57 dias a maxila contata o pré-frontal, nasal e articula-se com a pré-maxila. O aspecto trabecular permanece até a eclosão. Os centros de ossificação dos contra-laterais não se contatam até o fim da incubação (Figs 1-12).

Palatino. No embrião com 33 dias, o osso palatino está pouco corado. Aos 39 dias corresponde a uma pequena placa subtriangular que contata a maxila rostralmente e o pterigóide caudalmente aos 41 dias. No fim do período de incubação, a forma e a posição do palatino é um pouco diferente, mais largo e delimitando a margem medial da fenestra palatina (Figs 6-18).
Vômer. Aparece corado pela primeira vez como uma placa pequena e alongada, posicionada ventralmente ao septo interorbital e rostralmente aos ossos palatinos, ao nível do osso pré-frontal. Este elemento está presente no embrião de 33 dias de forma inconspícua. A pré-maxila, maxila e palatino estão topograficamente relacionados ao vômer e iniciam a ossificação antes deste. Numa fase inicial, as placas da pré-maxila, maxila e palatino estão marcadas com vermelho de alizarina e, durante a ontogenia, tais ossos se expandem e encobrem completamente ambas as placas do osso vômer na superfície palatal, permanecendo esta no assoalho da cavidade nasal. Aos 48 dias está posicionado à semelhança dos indivíduos adultos, encoberto pelos ossos pré-maxila e palatino, e não pode ser visualizado na superfície palatal do crânio.

Ectopterigoide. Está presente no embrião com 36 dias, embora pouco marcado com vermelho de alizarina, o que ocorre aos 42 dias, quando a ossificação avança e esboça o processo ascendente (Figs 1-18).

Pterigoide. Está presente como uma placa contínua, alongada e direcionada ventralmente no embrião de 33 dias. Estende-se nesta fase da porção posterior da fenestra palatina à porção posterior do crânio, na margem anterior da placa basal. São duas placas ventrais separadas por uma fenestra (precursor à da coana secundária) que se abre próximo à borda caudal da fenestra palatal. O centro de ossificação do pterigoide se estende ventrolateralmente ao longo da margem das trabéculas. Retém considerável quantidade de vermelho de alizarina, exceto lateralmente, formando uma asa no nível do jugal. Durante o desenvolvimento, a abertura da coana secundária migra caudalmente. Aos 51 dias a coana secundária está bem delimitada, e se posiciona próximo à margem posterior do pterigoide, no último estágio (Figs 1-18). Entretanto no animal adulto localiza-se na porção posterior do pterigoide. Durante as fases iniciais de desenvolvimento a placa pterigóide não contata a placa de substituição do quadrado, o que ocorre no último estágio, quanto a projeção dorsal do pterigoide contata a porção descendente do quadrado, aos 54 dias e bem ossificada delimitando a margem posterior do forame trigêmeo.

Jugal. Também é um dos primeiros a se ossificar. Forma uma estreita barra trirradiada na porção posterior da órbita aos 30 dias. Sua forma triangular é conspícua aos 33 dias, sendo a porção média do osso mais corada com vermelho de alizarina. Aos 39 dias contata a maxila, o quadradojugal e o osso pós-orbital. É posicionado lateralmente no crânio e ventroposteriormente na órbita, constituindo a margem rostral e ventral da fenestra temporal inferior (Figs 1-12).

Quadrado jugal. Aos 33 dias surge como uma pequena estrutura de forma trirradiada e pouco corada, posicionado rostralmente à cartilagem quadrada. Aos 39 dias está bem corado, com morfologia similar, embora este elemento aos 45 dias contate o jugal e o quadrado, mas não ainda o pós-orbital (Figs 1-12). 


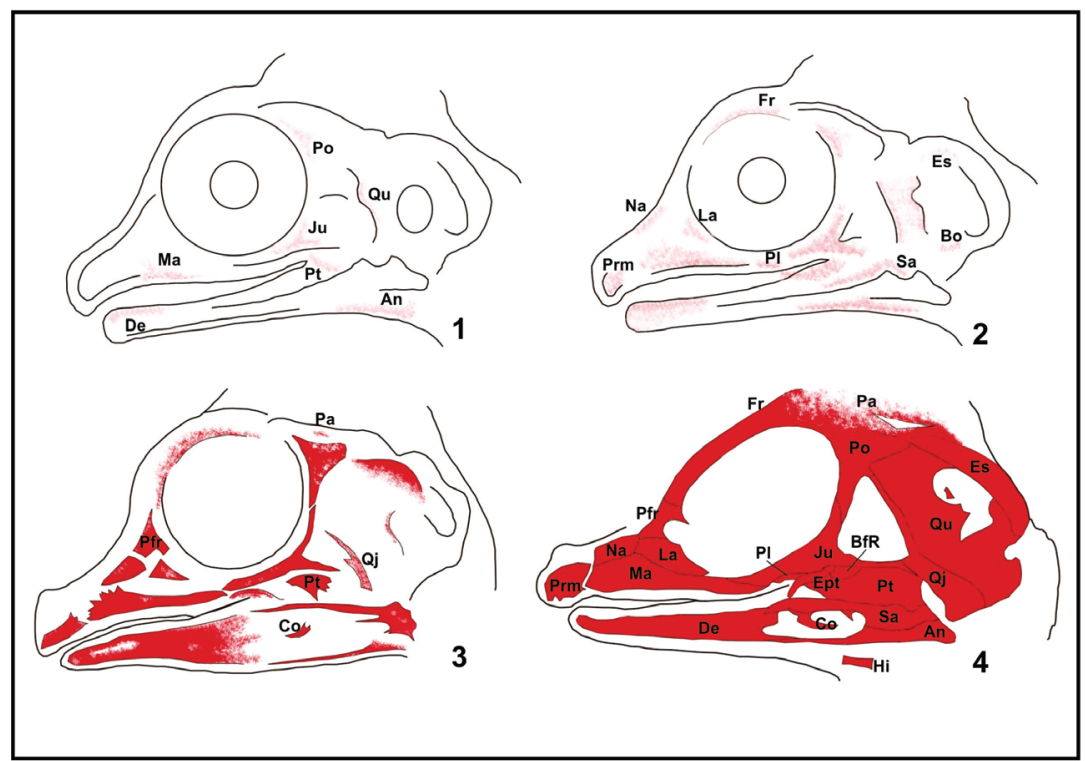

Figs. 1-4. Desenhos esquemáticos representativos das fases iniciais de desenvolvimento do crânio de Caiman yacare (Daudin, 1802), vista lateral: 1, 30 dias; 2, 33 dias; 3, 36 dias; 4, 39 dias (An, angular; Bo, basioccipital; BfR, basisfenoide rostral; Co, coronoide; De, dentário; Ept, ectopterigoide; Es, esquamosal; Fr, frontal; Hi, hioide; Ju, jugal; La, lacrimal; Ma, maxila; Na, nasal; Pa, parietal; Pfr, pré-frontal; Pl, palatino; Po, pós-orbital; Prm, pré-maxila; Pt, pterigoide; Qu, quadrado; Qj, quadradojugal; Sa, suprangular).

Nasal. O centro de ossificação do osso nasal é evidente aos 33 dias. Surge como uma placa independente sobre a placa parietotectal e se estende posteriormente sobre o septo nasal até a porção rostral do septo interorbital. Aos 39 dias sua morfologia está bem marcada com vermelho de alizarina. Contata a prémaxila, a maxila e o lacrimal aos 41 dias, e seu colateral aos 51 dias (Figs 1-12).

Lacrimal. O centro de ossificação do osso lacrimal é visualizado aos 33 dias na porção rostral da órbita óssea, lateralmente ao centro de ossificação para o osso pré-frontal. Com 36 dias apresenta considerável retenção de vermelho de alizarina e aspecto triangular conspícuo. Contata o nasal, maxila e pré-frontal aos 51 dias. Desenvolve um processo pontiagudo que se direciona caudalmente até a articulação entre a maxila e o jugal (Figs 1-12).

Pré-frontal. Posicionado rostralmente à órbita óssea, delimitando esta margem no crânio adulto. Nesta região da órbita é o primeiro a se corar com vermelho de alizarina, aos 33 dias. É relativamente robusto, posicionado na porção posterior do plano supraorbital e da comissura esfenoetimoide. Aos 43 dias apresenta o processo descendente desenvolvido, que se estende ao longo da borda posterior na passagem nasal, delimitando também a porção rostral da órbita. Com 51 dias é bem formado e contata os ossos nasal, lacrimal e frontal (Figs 1-12).

Frontal. É uma faixa estreita e pouco corada na margem rostral do crânio, medial às órbitas aos 36 dias de incubação. Os centros de ossificação (um por antímero) apresentam trabéculas ósseas que conferem aspecto esponjoso à placa, e com 36 dias estão pouco corados. Estes estendem-se do pré-frontal ao pós- orbital, e com 54 dias contatam o parietal. Os centros de ossificação de cada contra-lateral não se fundem no período embrionário, embora se contatem ao longo da linha mediana (Figs 1-18).

Pós-orbital. Junto com a maxila e o pós-orbital, ele é o primeiro a reter vermelho de alizarina aos 30 dias. Representa uma pequena placa triangular, que aos 33 dias contata o osso jugal. Seu esboço é bem corado em seus aspectos ventral e dorsal. Aos 51 dias, no contato com o osso parietal é trabecular (Figs 1-18).

Palpebral. É uma placa dérmica presente na superfície de cada membrana palpebral. Em $C$. yacare possui forma triangular e está posicionado na porção rostromedial da pálpebra, próximo à margem pré-frontal da órbita. Forma-se através de um centro de ossificação que se cora pela primeira vez aos 48 dias. Desenvolve-se rápido, estando conspícuo sobre o globo ocular aos 51 dias. Não contata com qualquer outro elemento ósseo (Figs 12, 17).

Esquamosal. Aparece no $33^{\circ}$ dia de incubação como um elemento triangular inconspícuo posicionado posterolateralmente no crânio. Está bem ossificado aos 45 dias. Ocupa a porção dorsocaudal da cápsula óptica, acima da cartilagem quadrada. Aos 39 dias contata o pós-orbital, o quadrado, o supraocciptal, o exoccipital e o parietal. Limita a porção laterocaudal da fenestra temporal superior (Figs 1-24).

Parietal. Apresenta discreta retenção de vermelho de alizarina aos 36 dias de incubação, mais tarde que a maior parte dos demais elementos dérmicos do crânio. É uma placa alongada lateralmente, disposta no teto craniano e posterior ao pós-orbital. Ainda retém um pouco de corante aos 48 dias. Cresce através de dois centros de ossificação que se fundem durante o 


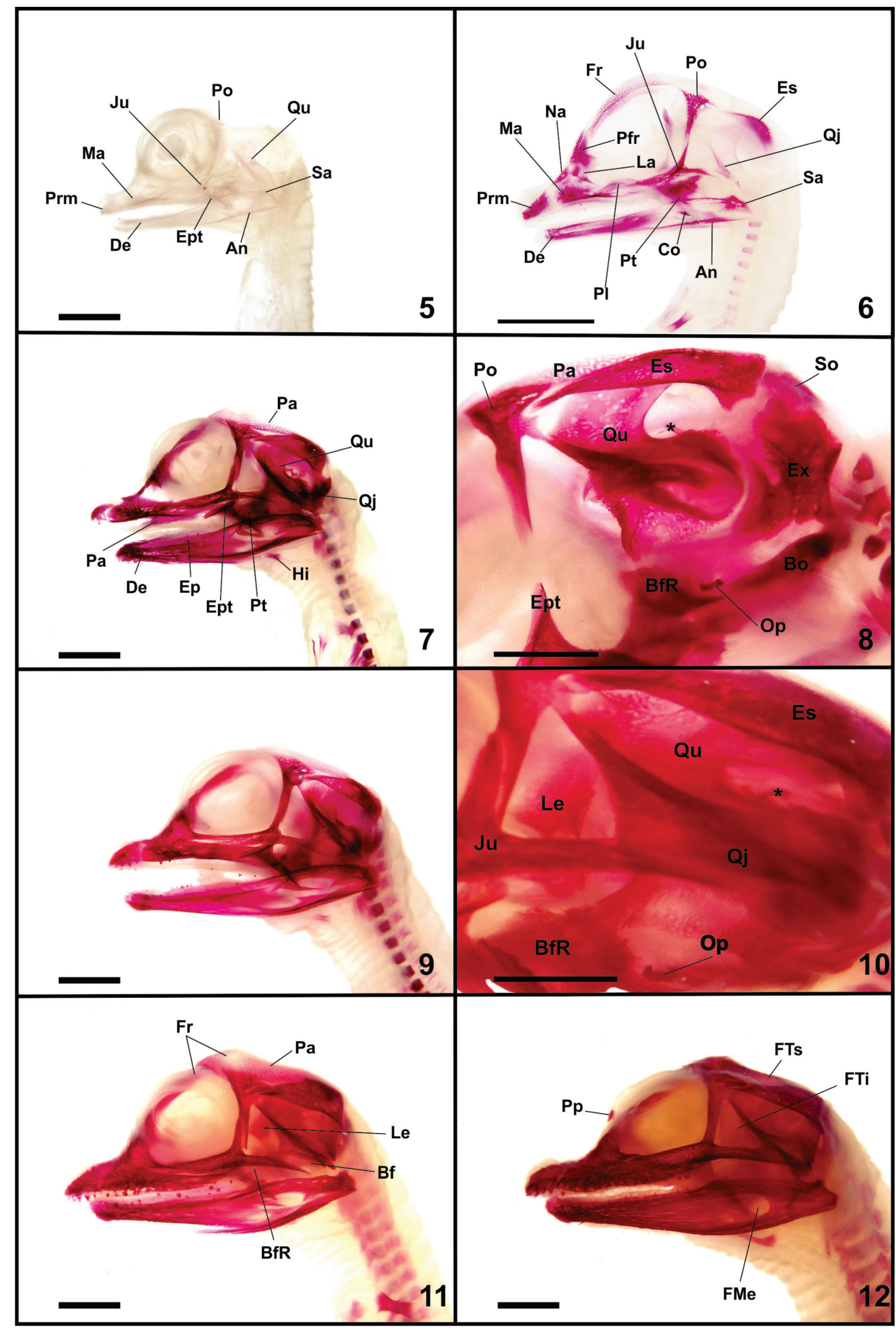

Figs. 5-12. Sincrânio de Caiman yacare (Daudin, 1802), vista lateral: 1, 33 dias; 2, 36 dias; 3, 39 dias; 4, detalhe da região látero-posterior aos 39 dias; 5, 45 dias; 6, detalhe da região látero-posterior aos 45 dias; 7, 54 dias e 8, 57 dias de incubação (An, angular; Bf, basisfenoide; BfR, basisfenoide rostral; Bo, basioccipital; Co, coronoide; De, dentário; Ept, ectopterigoide; Es, esquamosal; Ep, esplenial; Fr, frontal; FMe, fenestra mandibular; Fti, fenestra temporal inferior; Fts, fenestra temporal superior; Hi, hioide; Ju, jugal; La, lacrimal; Le, lateroesfenoide; Ma, maxila; $\mathrm{Na}$, nasal; Op, opistótico; Pa, parietal; Pfr, pré-frontal; Pl, palatino; Po, pós-orbital; Pp, palpebral; Pr, pró-ótico; Prm, pré-maxila; Pt, pterigoide; $\mathrm{Qu}$, quadrado; Qj, quadradojugal; Sa, suprangular; So, supraoccipital). Barra: $10 \mathrm{~mm}$, exceto figs 8 e 10, $3 \mathrm{~mm}$. 


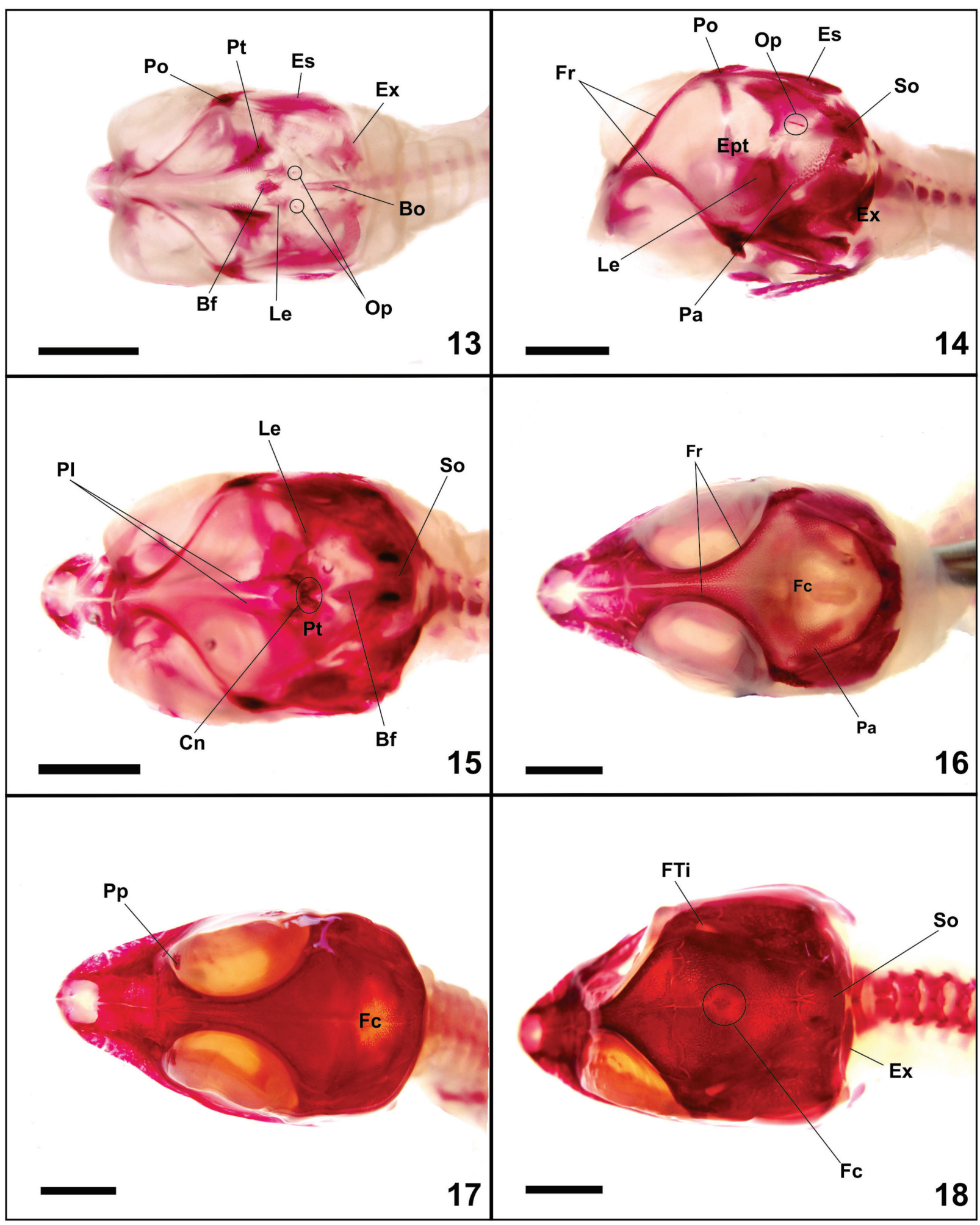

Figs. 13-18. Sincrânio de Caiman yacare (Daudin, 1802), vista dorsal: 13, 36 dias; 14 e 15, 39 dias; 16, 48 dias; 17, 51 dias; 18, 54 dias de incubação (Bf, basisfenoide; Bo, basioccipital; Cn, coana secundária; Eo, epi-ótico; Ept, ectopterigoide; Es, esquamosal; Ex, exocciptal; Fc, fontanela cranial; Fr, frontal; Fti, fenestra temporal inferior; Le, lateroesfenoide; Op, opistótico; Pa, parietal; Pl, palatino; Po, pós-orbital; Pp, palpebral; Pr, pró-ótico; Pt, pterigoide; So, supraoccipital). Barra: $10 \mathrm{~mm}$. 


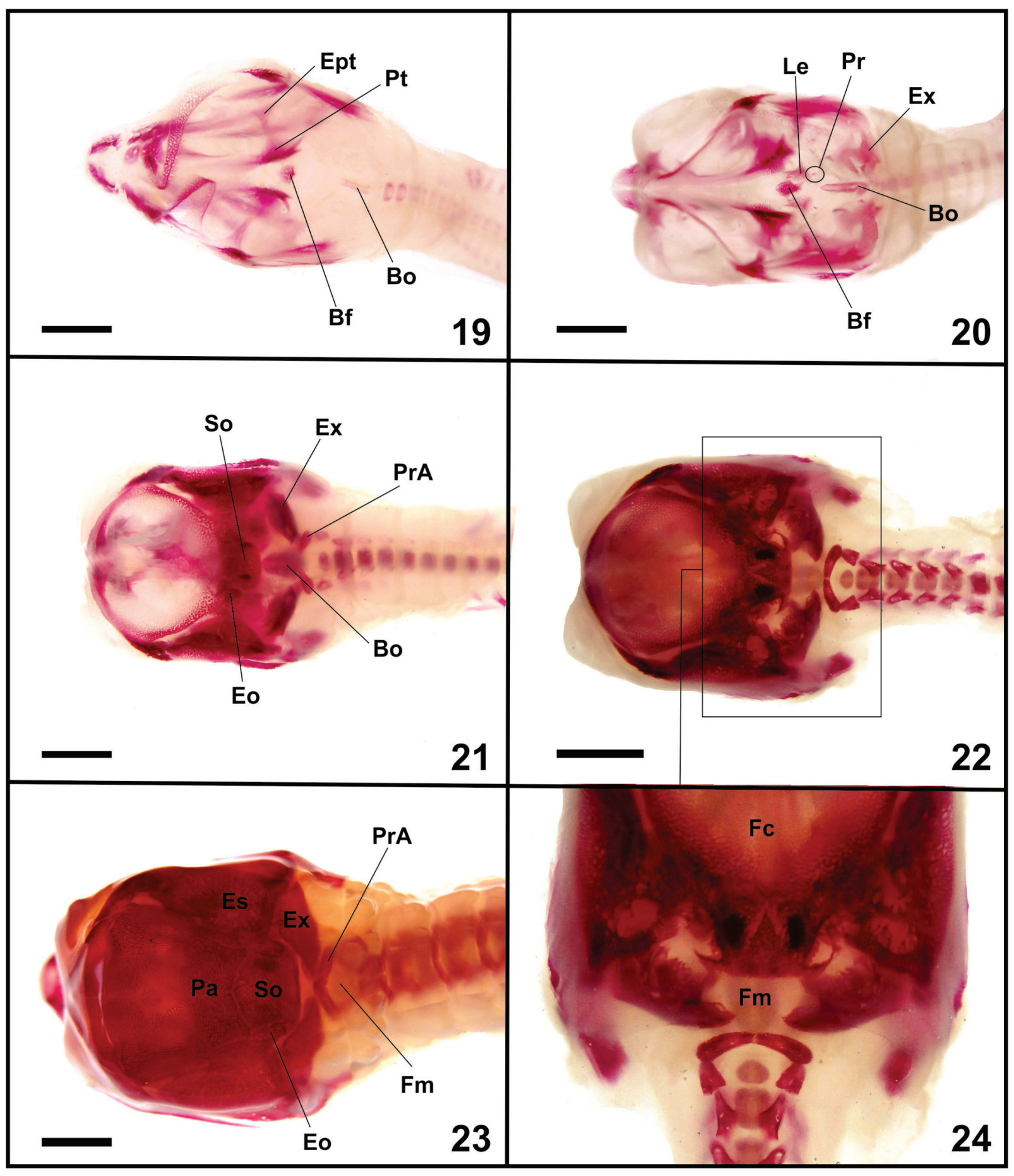

Figs. 19-24. Sincrânio de Caiman yacare (Daudin, 1802), vista caudal: 19, 36 dias; 20, 39 dias; 21, 45 dias; 22, 48 dias; 23, 54 dias; 24, detalhe da porção caudal aos 48 dias de incubação (Bf, basisfenoide; Bo, basioccipital; Eo, epi-ótico; Ept, ectopterigoide; Es, esquamosal; Ex, exocciptal; Fc, fontanela cranial; Fm, forame magno; Le, lateroesfenoide; Pa, parietal; Pr, pró-ótico; PrA, pró-atlas; Pt, pterigoide; So, supraoccipital). Barra: $10 \mathrm{~mm}$. 
desenvolvimento, aos 57 dias. Aos 51 dias o parietal está bem ossificado. Com 57 dias os centros de ossificação se expandem pelas porções caudal e medial, onde contatam seu colateral. Delimita a margem rostromedial da fenestra temporal superior (Figs 1-24).

Quadrado. Aos 36 dias a ossificação do quadrado inicia-se como uma pequena área corada que contorna a borda da incisura columelar. Aos 39 dias a coloração devido ao vermelho de alizarina é conspícua, mas os contornos da cartilagem permanecem. Aos 42 dias está fortemente corado. (Figs 1-12).

Basisfenoide. O basisfenoide é visível pela primeira vez no embrião de 33 dias como uma discreta placa na porção rostral da placa basal. Desenvolvese a partir de um único centro de ossificação que substitui parte da placa basal e a porção posterior das trabéculas. Aos 42 dias está bem ossificado e se expande lateralmente (Figs 13-24). O centro de ossificação do parasfenoide não foi observado. Este se funde ao basisfenoide durante a ontogenia (IORDANSKY, 1973), o que pode ter dificultado na sua identificação, além da presença de possíveis artefatos na técnica realizada.

Lateroesfenoide. Inicia a ossificação aos 36 dias. Aos 48 dias contata o quadrado e o pterigoide. É possível que sua posição, e do pterigoide, durante sua formação impeça a visualização da placa que origina o paraesfenoide, que em outros crocodilianos se ossifica naquela região (IoRDASNKY, 1973; Belairs \& KAMAL, 1981) (Figs 5-24).

Basioccipital. Se ossifica a partir de uma pequena placa que se cora aos 33 dias na porção posterior da placa basal. É alongado e posicionado ventralmente na porção posterior do crânio, projetando-se caudalmente durante o período de incubação. $\mathrm{O}$ côndilo occipital não se forma completamente antes do nascimento. Não contata nenhum outro osso até o $48^{\circ}$ dia (Figs 5-24).

Exoccipital. Surge no $39^{\circ}$ dia como um centro de ossificação alongado e lateral na porção caudal do crânio. Está bem corado, mas não se articula com qualquer outro elemento antes dos 51 dias, quando contata o supraoccipital e o esquamosal (Figs 13-24).

Pró-ótico. O pró-ótico está presente no embrião com 39 dias como de um centro de ossificação inconspícuo na porção ventrolateral do crânio, posteriormente ao laterosfenoide (Figs 5-18). Ossifica-se na cápsula óptica, junto à margem rostral da cartilagem quadrada e forma $\mathrm{a}$ margem caudal do forame para o nervo trigêmeo.

Epi-ótico. Está presente também aos 39 dias. Surge como uma pequena placa que se ossifica na margem lateral do osso supraoccipital, com qual se funde aos 51 dias (Figs 19-24).

Opistótico. É observado pela primeira vez no embrião com 39 dias. Apresenta inicialmente um centro de ossificação notável e bem corado na porção intracraniana. Outros centros de ossificação menos expressivos são notados na ontogenia, mas se fundem para formar um único osso robusto e pneumático aos 51 dias. Durante o desenvolvimento se relaciona topograficamente com a ossificação da porção distal da columela. No embrião com 45 dias o centro de ossificação da columela está próximo aos centros de ossificação do osso (Figs 13, 15), embora possa ser diferenciado destes devido a sua forma alongada (Figs 7,8).

Supraoccipital. Forma-se a partir de um único e estreito centro de ossificação localizado medialmente aos ossos epi-óticos no teto sinótico. Cora-se pela primeira vez aos 36 dias. Aos 51 dias ele se funde aos ossos epi-óticos (Figs 19-24).

Dentário. É um dos primeiros elementos a ossificar, indicado pela incipiente retenção de corante aos 30 dias. O centro de ossificação é alongado e se direciona ventrolateralmente na margem rostral da cartilagem de Meckel. A placa que forma o dentário se localiza na porção anterolateral da cartilagem de Meckel, cobrindo dois terços desta. No embrião com 33 dias o centro de ossificação está corado com vermelho de alizarina. Aos 36 dias o dentário cobre a porção anterior da cartilagem de Meckel e estende-se caudalmente ao longo da margem ventral desta onde contata o angular. Contata medialmente seu colateral aos 48 dias (Figs 1-12).

Esplenial. Apresenta fraca retenção de corante aos 33 dias na porção anterior da face medial da cartilagem de Meckel cobrindo dois terços da margem interna desta. Juntamente com o dentário, o esplenial cobre a cartilagem de Meckel e também se estende caudalmente ao longo desta (Figs 1, 2).

Suprangular. Aparece no embrião de 33 dias, embora pouco corado. O centro de ossificação possui forma de uma faixa estreita e alongada, localizada posteriormente a cartilagem de Meckel junto a área articular da mandíbula. Está bem corado aos 36 dias, quando assume uma forma triangular. Aos 42 dias articula-se com o dentário e o angular (Figs 1-12).

Coronóide. Está bem corado no embrião com 36 dias. É uma placa situada no limite do terço médio e posterior da cartilagem de Meckel, ao nível do processo ascendente do jugal. É bem desenvolvido no embrião com 42 dias na margem posterior do dentário (Figs 1-12).

Angular. O angular aparece no embrião de 33 dias. É uma placa estreita e pouco corada, posicionada ventromedialmente na porção caudal da cartilagem de Meckel, próximo à área articular. Possui um único centro de ossificação alongado que contata o dentário e o suprangular aos 42 dias (Figs 1-12).

Articular. O primeiro indício do osso articular é evidente no embrião com 39 dias, como uma pequena placa pouco corada medialmente ao suprangular. O articular se ossifica na superfície dorsal da porção posterior da cartilagem mandibular, imediatamente abaixo da extremidade inferior da cartilagem quadrada. Está bem corado aos 42 dias, quando se funde ao suprangular (Figs 1-12). 
Hioide. Se mantém junto à estrutura do crânio por meio de ligamentos e tendões. Sua ossificação começa aos 36 dias. A retenção de vermelho de alizarina avança pouco, mas é bem evidente aos 42 dias (Figs 4, 12-18).

\section{DISCUSSÃO}

Heterocronia, variação no desenvolvimento e padrão morfológico do esqueleto. A sequência de formação do esqueleto reflete a demanda funcional dos animais. A ossificação do crânio em anuros, por exemplo, atende as necessidades dos elementos envolvidos com a respiração (MABEE et al., 2000), e em peixes teleósteos, também com a alimentação, incluindo as espécies que apresentam estratégias peculiares de alimentação [e.g. Danio rerio (Hamilton, 1822)] seguidos por aqueles associados a suporte e depois aqueles de proteção (Weisel, 1967; Gaudin, 1978). Padrão similar é averiguado em C. yacare, que inicia sua ossificação pela maioria dos ossos da mandíbula, a maxila, a pré-maxila, os do palato e o jugal. A ossificação dos elementos da mandíbula ocorre mais cedo em grupos animais que possuem dentes e mais tarde em tetrápodes desdentados, podendo exemplificar uma cascata de desenvolvimento compartilhado que controla o início da formação do sincrânio (SCHOCH, 2006).

Nos répteis Archosauria, os elementos das mandíbulas e aqueles da órbita se ossificam primeiro, os do neurocrânio mais tarde. Em geral, nos crocodilianos, o palato se ossifica antes do neurocrânio, estando os pterigóides bem desenvolvidos em C. yacare já aos 42 dias. A maxila e também os ossos da mandíbula se desenvolvem cedo e rápido, padrão similar a $A$. mississippiensis (RIEPPEL, 1993; YeH, 2002). Em Lepidosauria, a sequência de ossificação do crânio se inicia pelo pterigóide (RiePPEL, 1992) e em C. palustris os primeiros ossos ossificam simultaneamente (a saber, frontais, parietais, pós-orbitais, esquamosais, pré-fontais, nasais, maxilas, pré-maxilas, palatinos, ectopterigoides, pterigoides, quadradojugais, jugais e vômer). Em $C$. porosus o paraesfenoide se ossifica por meio de três centros de ossificação, fundindo-se com o basisfenoide (Bellairs \& Kamal, 1981), todavia, em C. yacare o basisfenoide não foi observado durante a ossificação. A presença do paraesfenoide é uma característica dos Archosauria (IoRDASNKY, 1993), e está presente em $C$. yacare, como nos demais crocodilianos (e.g. C. palustris, C. porosus, C. niloticus, A. mississippiensis, A. sinensis, Caiman crocodilus Linnaeus, 1758); neste contexto, é possível que algum artefato na técnica ou o intervalo entre as coletas tenha inviabilizado a observação de seu desenvolvimento.

O latereosfenoide e o supraoccipital aparecem simultaneamente em C. palustres, C. porosus (Bellairs \& KAMAL, 1981) e em C. yacare, o lateroesfenoide inicia sua ossificação por meio de um centro de ossificação independente para cada osso, depois do basioccipital, basisfenoide e supraoccipital. O lateroesfenoide apresenta estrutura similar em crocodilianos e aves, além de alguns Archosauria extintos, o que reforça a relação evolutiva entre os Archosauria. Ele se ossifica na parede lateral do crânio, mas não é homólogo ao alisfenoide dos mamíferos (Bellairs \& Kamal, 1981).

Os ossos do crânio de C. yacare e de outros crocodilianos e dos Testudines, se desenvolvem a partir de trabéculas ósseas como em embriões de mamíferos e contrária à aparência compacta dos ossos dos Lepidosauria (Bellairs \& Kamal, 1981). A textura dos ossos em C. yacare reflete bem esta característica, principalmente nos ossos frontal, parietal, pós-orbital, maxila, pré-maxila, jugal e nos elementos mandibulares. Inferimos que esta característica possivelmente se relaciona com a formação de ossos mais leves, talvez uma característica compartilhada entre os Archosauria.

Caiman yacare compartilha com Gallus Brisson, 1760 e Alligator Daudin, 1809 aspectos do padrão de ossificação semelhante (e.g. início pela formação do jugal), sendo esta uma característica derivada compartilhada entre mamíferos, aves e crocodilianos ( $\mathrm{SCHOCH}, 2003)$, e por outros grupos extintos de tetrápodes (RIEPPEL, 1992). Análises ontogenéticas sugerem que, na maioria dos vertebrados estudados, pequenos grupos de ossos aparentemente deslocam-se em conjunto ao longo do processo evolutivo dos vertebrados, possivelmente em resposta à pressão do desenvolvimento do crânio (SCHOCH, 2006).

Em contrapartida, entre Squamata e Crocodylia a sequência de ossificação varia substancialmente. Em Lacerta agilis Linnaeus, 1758, o pterigoide, palatino, jugal e suprangular ossificam primeiro, seguidos pelos elementos da mandíbula (RIEPPeL, 1992). Em A. mississippiensis o pterigoide ossifica primeiro, seguido pelos ossos da mandíbula, maxila e pré-maxila e posteriormente pelos elementos do dermatocrânio. Caiman yacare tem padrão similar sendo que o nasal, o parietal os elementos endocondrais se ossificam tardiamente (De SÁ \& Trueb, 1991; Yeh, 2002).

O desenvolvimento do palato secundário nos Crocodylia segue paralelo à evolução do palato dérmico secundário nos crocodilianos fósseis (Geosaurus, Notosuchus, Sebecus, Trematochampsa) (ANDRADE et al., 2006). Continuando seu desenvolvimento, a coana apresenta gradual deslocamento posterior no pterigoide, o que progride durante a vida pós-embrionária nos crocodilianos (Kälin, 1933; LANGSTON, 1973; Ferguson, 1985). Embora ocorra a migração da coana secundária para a porção caudal do pterigoide nos crocodilianos jovens (KäLIN, 1933), em Caiman esta tendência aparece ainda durante a vida embrionária (MONTEIRO \& SoARES, 1997). Também a migração da região da coana secundária ocorre ainda durante os primeiros eventos ontogênicos, haja vista que os pterigoides se desenvolvem precocemente neste espécie.

A sutura entre os pterigoides se forma muito 
tempo após o nascimento em $A$. mississippiensis, mas é evidente em C. yacare já no período pré-eclosão. Em alguns crocodilianos adultos os pterigoides e os palatinos são dilatados, o que provavelmente reflete uma condição pneumática no crescimento do ducto nasofaringeal, como ocorre em gaviais (que representa o caso mais conhecido desta particularidade) (IORDANSKY, 1973).

Segundo ANDRADE et al. (2006), a morfologia do palato e narinas internas dos Crocodylomorpha é importante para a sistemática deste grupo, sendo sua classificação baseada em estudos acerca das características gerais e do desenvolvimento destas estruturas (ROMER, 1956; IORDANSKY, 1973; BENTON \& Clark, 1988; Carroll, 1988; Brochu, 2003; Pough et al., 2003; Hutchinson, 2006). A abertura nasal de $C$. yacare é única, diferente de Alligator e Osteolaemus onde é septada pelo pequeno processo pré-nasal ascendente da maxila que se encontra com a extensão anterior dos ossos nasais e divide a abertura nasal (BELLAIRS \& KamaL, 1981).

Em $C$. yacare e $M$. niger os ossos que compõem a mandíbula e aqueles da porção rostral do crânio se alongam pouco no período de incubação, sendo o alongamento do focinho mais pronunciado no início da vida adulta. Com base nos resultados apresentados para $C$. yacare, é possível inferir que a sequência de ossificação atende à demanda funcional dos animais, outrora, os processos morfogênicos que envolvem o alongamento do crânio sejam uma característica plesiomórfica (MonTEIRo \& SOARES, 1997). IORDANSKY (1973) relata que algumas variações no crânio de $C$. paulistris, $C$. porosus e C. niloticus (principalmente na porção rostral), estão relacionadas à alimentação e à ação de músculos mandibulares (além do dimorfismo sexual, este ainda não pormenorizado). De fato, os ossos que compõem a porção rostral do crânio de C. yacare se ossificam nos primeiros estágios de desenvolvimento, o que confirma em parte as informações fornecidas pelos autores acima citados. Todavia são necessários subsídios sobre o processo de formação dos músculos mandibulares - bem como suas relações funcionais com o arcabouço ósseo da região rostral do crânio -, para confirmar em plenitude esta hipótese.

$\mathrm{Na}$ porção posterior do crânio de C. yacare, similar a A. mississpipiensis, o basioccipital é o primeiro a se ossificar, seguido pelo basisfenoide, exoccipital e opistótico, pela porção dorsal do epiótico. Em ambos os ossos epiótico e pró-ótico são indiferenciáveis (fundidos respectivamente com o parietal e lateresfenoide), embora se ossifiquem de forma independente. Segundo IORDANSKY (1973), o opistótico está fundido com o exoccipital e talvez por este motivo é dificilmente distinguível durante a ontogenia do esqueleto, mas em C. yacare ele se forma a partir de centros de ossificação diversos, alguns bem evidentes e outros nem tanto, para cada placa óssea. Com exceção do exoccipital, estes ossos são de substituição do crânio cartilaginoso e geralmente se formam em estágios finais de desenvolvimento nos vertebrados.

Segundo Schoch (2006), os ossos que formam o teto do crânio (esquamosal, parietal e frontal) se ossificam todos ao mesmo tempo nos répteis. Este padrão é diferente em C. yacare e A. mississippiensis (RIEPPEL, 1993), onde o parietal se ossifica depois dos demais e a partir de dois centros de ossificação distintos. A porção posterior da órbita óssea em ambos os crocodilianos está completamente fechada neste estágio, outrora o teto dérmico da caixa craniana permanece aberto, e a separação dos ossos frontais bem marcada.

Sphenodon punctatus (Gray, 1842), L. agilis e Zooteca vivipara (von Jacquin, 1787) diferente de $C$. yacare e Alligator (RIEPPEL, 1992) em alguns aspectos da sequência de ossificação. Dentre estas diferenças destacamos a ossificação do parietal em Lepidosauria (que não é um dos últimos a se ossificar) e a ossificação do pterigoide em L. agilis (que ocorre no início do período de incubação, juntamente com o palatino e o jugal). A diferença cronológica na ossificação do pterigoide e dos ossos palatais entre L. agilis, C. yacare e A. mississippiensis pode estar ligada às alterações da presença da coana secundária em crocodilianos.

O esquamosal em $C$. yacare e $A$. mississippiensis começa sua ossificação seguindo a margem posterior do teto craniano. Este avança e delineia, juntamente com o parietal, a forma da fenestra temporal superior e a grande fontanela cranial, que se fecha apenas no período pós-natal (RIEPPEL, 1993). Tal fontanela também está presente em algumas espécies de peixes, onde ela também reduz gradativamente seu tamanho durante a embriogênese (Adriaens \& Verraes, 1998).

Segundo Mabee \& Trendler (1996), grande parte das alterações na sequência de ossificação podem simplesmente refletir alterações genéticas entre as espécies. Outrora, podemos ainda inferir que tais mudanças podem ser condicionadas através da evolução devido às particularidades de cada grupo. Existem também variações desencadeadas por fatores ambientais, funcionais e comportamentais que influenciam no desenvolvimento de determinados caracteres (HERRING, 1993), o que possivelmente explica as variações de padrão encontradas entre os diferentes vertebrados descritos e C. yacare.

\section{REFERÊNCIAS BIBLIOGRÁFICAS}

Adriaens, D. \& Verraes, W. 1998. Ontogeny of the Osteocranium in the African Catfish, Clarias gariepinus Burchell (1822) (Siluriformes: Clariidae): ossification sequence as a response to functional demands. Journal of Morphology 235(3):183-237.

Alberch, P.; Gould, S. J.; Oster, G. F. \& Wake, D. B. 1979. Size and shape in ontogeny and phylogeny. Paleobiology 5:296-317.

Andrade, M. B.; Bertini, R. J. \& Pinheiro, A. E. P. 2006. Observations on the palate and choannae structures in Mesoeucrocodylia (Archosauria, Crocodylomorpha): phylogenetic implications. Revista Brasileira de Paleontologia 9(3):323-332.

Bellairs, A. d'A. 1987. The crocodilia. In: WeBb, G. J. W.; Manolis, S. C. \& Whitehead, P. J. eds. Wildlife Management: crocodiles and alligators. Chiping Norton, Surrey Beatty and Sons. p. 5-7. 
Bellairs, A. D'A. \& Kamal, A. M. 1991. The chondrochanium and the development of the skull in recent reptiles. In: GANs, C. \& Parsons, T. S. eds. Biology of the Reptilia. London, Academic. v.11, 263p.

Benton, M. J. \& Clark, J. M. 1988. Archosaur phylogeny and the relationships of the Crocodylia. In: Benton, M. J. ed. The Phylogeny and Classification of the Tetrapods: Amphibians, Reptiles, Birds. Oxford, Clarendon Press. v. 1, p.295-338.

Brochu, C. A. 2003. Phylogenetic approaches toward crocodylian history. Annual Review of Earth and Planetary Sciences 31:357-397.

BuRKe, C. A. 1989. Development of the turtle carapace: implications for the evolution of a novel Bauplan. Journal of Morphology 199(3):363-378.

CARroll, R. L. 1988. Vertebrate Paleontology and Evolution. New York, Freeman. 698p.

Davis, D. D. \& Gore, U. R. 1936. Clearing and staining skeleton of small vertebrates. Field Museum of Natural History 4:3-15.

De Beer, G. R. 1937. The Development of the Vertebrate Skull. Oxford, Oxford University Press. 552p.

DE SÁ, R. O. 1988. Chondrocranium and ossification sequence of Hyla lanciformis. Journal of Morphology 195(3):345-355.

De SÁ, R. O. \& Trueb, L. 1991. Osteology, skeletal development and chondrocranial structure of Hamptophryne boliviana (Anura: Microhylidae). Journal of Morphology 209(3):311-330.

Dodson, P. 1975. Functional and ecological significance of relative growth in Alligator mississippiensis. Journal of Zoology 175:315-355

FERguson, M. W. J. 1985. Reproductive biology and embryology of the crocodilians. In: Gans, C.; Billett, F. \& Maderson, P. F. A. eds. Biology of the Reptilia. London, Academic. v.5, p.329-491.

1987. Post-laying stages of embryonic development in crocodilians. In: WeBB, G. J. W.; Manolis, S. C. \& Whitehead, P. J. eds. Wildlife management: crocodiles and alligators. Chipping Norton, Surrey Beatty \& Sons. p.427-444

Gans, C. \& Northcutt, R. G. 1983. Neural crest and the origin of vertebrates: a new head. Science 220(4594):268-274.

Gaudin, A. J. 1978. The sequence of cranial ossification in the California Toad, Bufo boreas (Amphibia, Anura, Bufonidae). Journal of Herpetology 12(2):309-318.

Hanken, J. \& Hall, B. K. 1988. Skull development during anuran metamorphosis: I. Early development of the first three bones to form - the exoccipital, the parasphenoid, and the frontoparietal. Journal of Morphology 195(3):247-256.

Herring, S. W. 1993. Epigenetic and functional influence in skull growth. In: Hanken, J. \& Hall, B. K. eds. The Skull. Chicago, University Chicago. v.1, p.153-206.

Hickman, C. P. JR.; Roberts, L. S.; Larson, A.; I'anson, H. \& Eisenhour, D. J. 2006. Amniote origens and reptilian groups. In: Hickman, C. P. JR. ed. Integrated Principles of Zoology. New York, Higher Education. p.547-567.

Hutchinson, J. R. 2006. The evolution of locomotion in archosaurs. Comptes Rendus Palevol 5(3-4):519-530.

IORDANSKY, N. N. 1973. The skull of the crocodilian. In: Gans, C. \& PARsons, T. S. eds. Biology of the Reptilia. London, Academic. v.4, p. 201-264.

IUngman, J.; Piña, C. I. \& Siroski, P. 2008. Embryological development of Caiman latirostris (Crocodylia: Alligatoridae). Genesis 46(8):401-417.

Kälın, J. A. 1933. Beiträge zur vergleichenden Osteologie des Crocodilienschädels. Zoologische Jahrbücher 57:535-714

KLEMBARA J. 1991. The cranial anatomy of early ontogenetic stages of Alligator mississippiensis (Daudin, 1802) and the significance of some of its cranial structures for the evolution of tetrapods. Palaeontograph Abteilung A: Paleaozoologie 215:103-171.

. 2004. Ontogeny of the palatoquadrate and adjacent lateral cranial wall of the endocranium in prehatching Alligator mississippiensis (Archosauria: Crocodylia). Journal of Morphology 262:644-658.

2005 . Ontogeny of the partial secondary wall of the otoccipital region of the endocranium in prehatching Alligator mississippiensis (Archosauria, Crocodylia). Journal of Morphology 266:319339.
Langille, R. M. \& Hall, B. K. 1987. Development of the head skeleton of the Japanase Medaka, Oryzias latipes (Teleostei). Journal of Morphology 193(2):135-158.

LANGSTON, W. 1973. The crocodilian skull in historical perspective. In: Gans, C. \& Parsons, T. S. eds. Biology of the Reptilia. London, Academic. v.4, p.265-284.

Mabee, P. M. \& Trendler T. A. 1996. Development of the cranium and paired fins in Betta splendens (Teleostei: Percomorpha) intraspecific variation and interspecific comparations. Journal of Morphology 227(3):249-287.

Mabee, P. M.; Otmstead, K. L. \& Cubbage, C. C. 2000. An experimental study of intraspecific variation, developmental, timing, and heterochrony in fishes. Evolution 54(6):2091-2106.

Mader, D. R. 2006. Reptile Medicine and Surgery. Saint Louis, Saunders Elsevier. 658p.

Magnusson, W. E. \& TAYlor, J. A. 1980. A description of developmental stages in Crocodylus porosus, for use in adding eggs in the field. Australian Wildlife Research 7:479-485.

Meek, A. 1911. On the morphogenesis of the head of the crocodile. Journal Anatomy Physiology 45:357-377.

Meyer, A. \& Zardoya, R. 2003. Recent advances in the (molecular) phylogeny of vertebrates. Annual Review of Ecology and Systematics 34:311-338

Monteiro, L. R. \& Soares, M. 1997. Allometric analysis of the ontogenetic variation and evolution of the skull in Caiman Spix, 1825 (Crocodylia: Alligatoridae). Herpetologica 53(1):62-69.

Monteiro, L. R.; Cavalcanti, M. J. \& Sommer III, H. J. S. 1997. Comparative ontogenetic shape changes in the skull of Caiman species (Crocodylia, Alligatoridae). Journal of Morphology 231(1):53-62.

Моoк, C. C. 1921. Skull characters of recent Crocodilia, with notes on the affinities of recent genera. Bulletin of the American Museum of Natural History 44(13):123-268.

1923. Skull characters of Alligator sinensis Fauvel. Bulletin of American Museum of Natural History 48(16):553-562.

Müller, G. B. \& Alberch, P. 1990. Ontogeny of the limb skeleton in Alligator mississippiensis: developmental invariance and change in the evolution of Archosaur limbs. Journal of Morphology 203(2): 151-164.

MüLLER, J. 2003. Early loss and multiple return of the lower temporal arcade in diapsid reptiles. Naturwissenschaften 90:473-476.

NAISH, D. 2001. Fossils explained: Crocodilians. Geology Today 17(2):71-77.

NorthCuTt, R. G. \& Gans, C. 1983. The genesis of the neural crest and epidermal placodes: a reinterpretation of vertebral origins. The Quarterly Review of Biology 58(1):1-28.

PARKER, W. K. 1882. On the structure and development of the skull in the Crocodile. Nature 26:252-254.

1883. On the structure and development of the skull in the Crocodilia. The Transactions of Zoological Society of London 26:252-254.

Pough, F. H.; Janis, C. M. \& Heiser, J. B. 2003. A Vida dos Vertebrados. São Paulo, Atheneu. 699p.

Richtsmeier, J. T.; Corner, B. D.; Grausz, H. M.; Cheverud, J. M. \& DanaheY, S. E. 1993. The role of postnatal growth in the crab-eating macaque (Macaca fascicularis). Journal Human Evolution 25:1-30.

Rieppel, O. 1992. Studies of formation in reptiles III. Patterns of ossification in the skeleton of Lacerta vivipara, Jacquin (Reptilia, Squamata). Fieldiana Zoologica 68:1-25.

1993. Studies of skeleton formation in reptiles. V. Patterns of ossification in the skeleton of Alligator mississippiensis Daudin (Reptilia, Crocodylia). Zoological Journal of the Linnean Society 109(3):301-325.

1995. Studies on skeleton formation in reptiles: implications for turtle relationships. Zoology 98:298-308.

2004. Kontroversen innerhalb der Tetrapoda - Die Stellung der Schildkröten (Testudines). Sitzungsberichte der Gesellschaft Naturforschender Freunde zu Berlin 43:201-221.

Rieppel, O. \& de Braga, M. 1996. Turtles as diapsid reptiles. Nature 384(6608):453-455.

Rieppel, O. \& Reisz, R. R. 1999. The origin and early evolution of Turtles. Annual Review of Ecology and Systematics 30:1-22. 
Romer, A. S. 1956. Osteology of the Reptiles. Chicago, Chicago University, $468 \mathrm{p}$.

SBH. Lista brasileira de répteis. 2010. Disponível em: <http:// www.sbherpetologia.org.br/checklist/repteis.htm>. Acesso em: 26.09.2010.

Sсносн, R. R. 2003. Early larval ontogeny of the Permo-Carboniferous temnospongyl Sclerocephalus. Palaeontology 46(5):1055-1072.

2006. Skull ontogeny: developmental patterns of fishes conserved across major tetrapod clades. Evolution \& Development 8(6):524-536.

SHeIL, C. A. 2005. Skeletal development of Macrochelys temminckii (Reptilia: Testudines: Chelydridae). Journal of Morphology 263(1):71-106.
SHirNo K. 1914. Studien zue Kenntnis des Wirbeltierkopfes. I. Das Chondrocranium von Crocodilus mit Berücksichtigung der Gehirnnerven und Kopfgefasse. Anatomical Hefte 50:254-381.

Weisel, G. F. 1967. Early ossification in the skeleton of the sucker (Catostomus macrocheilus) and the guppy (Poecilia reticulate). Journal of Morphology 121(1):1-18.

Werneburg, I. \& Sánchez-Villagra, M. R. 2009. Timing of organogenesis support basal position of turtles in the amniote tree of life. Evolutionary Biology 9:82.

YeH, J. 2002. The evolution of development: Two portraits of skull ossification in pipoid frogs. Evolution 56(12):2484-2498. 